\title{
EVOLUÇÃO NO ATENDIMENTO \\ ODONTOLÓGICO DE PACIENTES COM \\ TRANSTORNO DO ESPECTRO DO AUTISMO \\ POR MEIO DE PECS
}

DOI: http://dx.doi.org/10.18616/prat04

\begin{abstract}
Andressa Martins da Costa
UNESC -Universidade do Extremo Sul Catarinense andressamartinscosta@outlook.com
\end{abstract}

Gabriela Aguiar Giassi UNESC - Universidade do Extremo Sul Catarinense gabriela.ag@live.com

Fernanda G. F. Sonego UNESC - Universidade do Extremo Sul Catarinense fgfsonego@unesc.net

\section{Renan Antonio Ceretta}

UNESC - Universidade do Extremo Sul Catarinense rce@unesc.net 
O autismo teve sua descoberta pelo psicólogo americano infantil Leo Kammer em 1943. Seu estudo teve como base a avaliação do comportamento de onze crianças que ele notou serem diferenciadas, principalmente no desenvolvimento social (DIAS, 2015).

O Transtorno do Espectro do Autismo (TEA) é classificado dentro de uma série de transtornos invasivos ao desenvolvimento, que normalmente está associado à deficiência intelectual (JAGANATHAN, 2014). É considerado um transtorno global do desenvolvimento anormal ou alterado, que normalmente se manifesta antes dos três anos de idade, onde o indivíduo acometido apresenta uma disfunção das interações sociais, habilidades físicas e comunicação bem como comportamento focalizado e repetitivo. Além disso, é acompanhado de diversos sintomas como fobias, perturbações de sono ou da alimentação, crises de birra ou até mesmo, em alguns casos mais severos, agressividade ou autoagressividade (ORGANIZAÇÃO MUNDIAL DE SAÚDE, 2008).

Dados de 2012 apontam que uma a cada sessenta e oito crianças nascidas possuem TEA e, dentro desta estatística, destaca-se maior prevalência no sexo masculino, acometendo um a cada quarenta e dois meninos (CHRISTENSEN et al., 2016).

Quanto às alterações faciais e bucais do paciente com TEA, pode-se verificar uma assimetria facial, associada a uma má oclusão, que necessita de tratamento ortodôntico corretivo. Além destas alterações eles possuem o desenvolvimento do sistema psicomotor afetado, o que dificulta a higiene oral, e leva ao acúmulo de tártaro nos dentes e a gengivite. Em um grande número de casos há presença de úlceras traumáticas, por hábitos lesivos (ORIQUI, 2006).

No atendimento odontológico direcionado a pacientes especiais, o cirurgião-dentista deve redobrar seu cuidado com o paciente e sua família, contando com a ajuda de uma equipe transdisciplinar para a resolução dos problemas (ALVES, 2004). Há casos em que o atendimento odontológico é realizado por meio de anestesia geral e/ou estabilização protetora (ZINK, 2012a). 
O Condicionamento Lúdico utiliza o método Son-Rise ${ }^{\varpi}$ e o Sistema de Comunicação por Intercâmbio de Figuras (PECS) na Odontologia. O método consiste em atrair o contato visual das crianças autistas, atuando como um manual para o treinamento das habilidades de comunicação interpessoal. Este sistema funciona por meio de imagens que descrevem o dia a dia e o tratamento dentro do consultório odontológico, proporcionando um tratamento mais humanizado, sem uso de estabilização protetora (ZINK, 2012b).

Essa pesquisa buscou avaliar o condicionamento lúdico no atendimento odontológico de crianças com diferentes graus de TEA, por meio do método Son-Rise ${ }^{\oplus}$ e Sistema de Comunicação por Intercâmbio de Figuras (PECS) adaptada para à Odontologia.

\section{MÉTODOS}

Tratou-se de uma série de casos, cuja análise foi descritiva. Foi realizado na Clínica de Odontologia na Universidade do Extremo Sul Catarinense (UNESC), no período de setembro de 2015 a abril de 2017, dividiu-se em duas etapas, onde os procedimentos clínicos durante a primeira etapa do estudo duraram seis meses e na segunda etapa três meses. A pesquisa foi aprovada pelo Comitê de Ética do local onde foi realizada a pesquisa sob o parecer n. 1.696.955/2016.

A população deste estudo foi composta de duas crianças portadoras de diferentes graus de TEA, que frequentavam a Associação Amigo dos Autistas - AMA de uma cidade no Sul Catarinense, com idade entre cinco e doze anos, sem vínculo familiar com o pesquisador que realizou o atendimento, e sem que já houvesse participado de um condicionamento lúdico para atendimento odontológico previamente ao estudo. Foi apresentado aos responsáveis o Termo de Consentimento Livre Esclarecido, que seguiu os princípios éticos contidos na Declaração de Helsinque e na resolução n. 466/12 do Conselho Nacional de Saúde. 
Em um primeiro momento, foi realizada anamnese com os pais e/ ou responsáveis, na qual foram obtidas as preferências das crianças, condições de saúde, experiências odontológicas e formas de abordagens para o desenvolvimento das atividades. Na sequência foram confeccionadas dez figuras (modelo PECS) adaptadas à Odontologia, que foram baseadas em um estudo já realizado (ZINK, 2012b).

Foi criada em forma de revista uma adaptação do PECS para um dos participantes, onde as imagens não foram modificadas, apenas sua forma de apresentação, as quais eram as mesmas contidas nas placas individuais da metodologia do estudo que serviu como base para este (ZINK, 2012b).

Cada processo do condicionamento lúdico foi repetido até que o paciente estivesse apto a realizar o novo conhecimento. $\mathrm{O}$ ambiente clínico foi isento de ruídos ou de qualquer atividade que propiciasse distração. Reforçadores positivos fizeram parte de todo o atendimento, tendo como objetivo a valorização de cada novo aprendizado. $\mathrm{O}$ atendimento odontológico contou com a presença de um auxiliar e um familiar, sendo que os mesmos foram orientados a não interferirem durante as sessões.

À medida que os pacientes foram condicionados, o grau de complexidade dos procedimentos odontológicos foi aumentado na mesma proporção. Caso houvesse a necessidade de algum procedimento mais complexo na primeira etapa do estudo, os pacientes seriam encaminhados à disciplina de Pacientes com Necessidades Especiais da instituição de ensino superior.

Os pacientes que obtiveram resultados positivos ao condicionamento lúdico foram incluídos na segunda etapa, que constituía acompanhamento e atendimento após o período de seis meses do fim da primeira etapa, nas Clínicas Integradas da UNESC, pelas mesmas pesquisadoras para a realização dos tratamentos odontológicos necessários com o uso ou não de anestesia local.

Os procedimentos clínicos realizados durante a primeira e a segunda etapa do estudo incluíram: profilaxia com o uso de ultrassom Gnatus ${ }^{\circledR}$, Curetas Gracey Quinelato ${ }^{\circledR}$, pasta profilática Maquira ${ }^{\circledR}$ associada à pedra-pomes, radiografias interproximais e selamento de fossas e fissuras com ionômero de vidro 
Ketac Molar Easy Mix da $3 \mathrm{M}^{\circledast}$, sempre acompanhados da técnica do falar-mostrar-fazer. Os Equipamentos de Proteção Individual (EPIs) como, touca $\mathrm{Fava}^{\oplus}$, máscara Fava ${ }^{\oplus}$, óculos de proteção e luva de procedimento Supermax ${ }^{\circledast}$ foram utilizados em todas as sessões de atendimento.

O estudo foi baseado nos métodos e técnicas utilizadas e desenvolvidas em uma pesquisa no estado de São Paulo ${ }^{8}$. Os dados obtidos em cada sessão foram documentados nos prontuários individuais desenvolvidos pelas pesquisadoras para o estudo.

\section{RESULTADOS}

Duas crianças com TEA foram selecionadas previamente, uma destas foi excluída, pois já havia sido condicionada anteriormente por outro profissional e o estudo requeria a inclusão apenas de crianças não condicionadas. Desta forma, outro participante foi selecionado.

Um dos participantes era do gênero feminino, 10 anos, com diagnóstico do CID 84.0 (Autismo infantil) que no estudo foi denominado como "F". Segundo anamnese, "F" apresenta anomalias na família de deficiência mental. Não foram relatadas alterações de saúde no último ano, apenas hospitalizações devido a crises convulsivas. Possui rinite alérgica, aftas e tosses frequentes, dificuldade de cuspir, problemas de visão e o hábito de ranger os dentes. Faz uso de Depakene $50 \mathrm{mg} / \mathrm{ml}$, que é uma medicação anticonvulsivante. Já realizou visita ao cirurgião-dentista para escovação supervisionada, porém os responsáveis relataram que foi uma experiência desagradável. A higiene bucal é realizada três vezes ao dia, por quem estiver em casa no horário (Tabela1).

A paciente " $F$ " já utilizou o sistema PECS para outros fins na escola regular e na AMA de Criciúma - Santa Catarina (SC). Os itens de que "F" mais gosta, relatados na anamnese, são desenho animado, músicas, massinhas de modelar, balão, touca e bolinhas de sabão. Possui aversão a barulhos excessivos e motos (Tabela 1). 
O outro participante era do gênero masculino, 10 anos, portador do CID 10 F79.0 (Retardo mental não especificado), F84.8 (Outros transtornos globais do desenvolvimento) mais G40.2 (Epilepsia e síndromes epilépticas). O TEA foi determinado por meio de diagnóstico clínico médico. No estudo foi denominado de "M". Segundo anamnese, não apresenta anomalias na família. No último ano teve quadros de gripe e foi hospitalizado devido à apendicite. Possui anemia, suspeita de problemas de visão, dificuldade de cuspir e range os dentes no período diurno (Tabela 1).

Faz uso das seguintes medicações: Neuleptil ${ }^{\circledR} 40 \mathrm{mg} / \mathrm{ml}$, que é uma medicação utilizada para a melhora de distúrbios do comportamento e, Carbamazepina ${ }^{\circledR} 20 \mathrm{mg} / \mathrm{ml}$, usada como medicação anticonvulsivante. Já realizou tratamentos odontológicos anteriores por meio de estabilização protetora e anestesia geral. $\mathrm{O}$ atendimento foi interrompido devido à dificuldade de colaboração pelo paciente, tornando os momentos desagradáveis e traumáticos no consultório odontológico. A escovação dental é realizada duas vezes ao dia, pela mãe (Tabela 1).

O paciente "M" já utilizou o sistema PECS na AMA de Criciúma-SC. Os itens de que " $\mathrm{M}$ " mais gosta, conforme o que foi relatado na anamnese, são livros ilustrados, desenhos animados, desenhar, bolinhas de sabão, ações no celular e se olhar no espelho. Possui aversão a barulhos excessivos, trajes brancos e EPIs, como toucas, máscaras e luvas (Tabela 1).

Tabela 1 - Perfil dos pacientes estudados na pesquisa. Criciúma, Brasil, 2016.

\begin{tabular}{c|c|c}
\hline \multicolumn{2}{|c|}{ Paciente "F" } & Paciente “M" \\
\hline Idade & 10 anos & 10 anos \\
CID & F840 & F790, F848, G402 \\
\hline Alterações de saúde & $\begin{array}{c}\text { Rinite alérgica, aftas e tosse } \\
\text { frequentes, dificuldade de } \\
\text { cuspir, problemas de visão e } \\
\text { o hábito de ranger os dentes }\end{array}$ & $\begin{array}{c}\text { Anemia, suspeita de } \\
\text { problemas de visão, } \\
\text { dificuldade de cuspir } \\
\text { e range os dentes no } \\
\text { período diurno }\end{array}$ \\
\hline
\end{tabular}




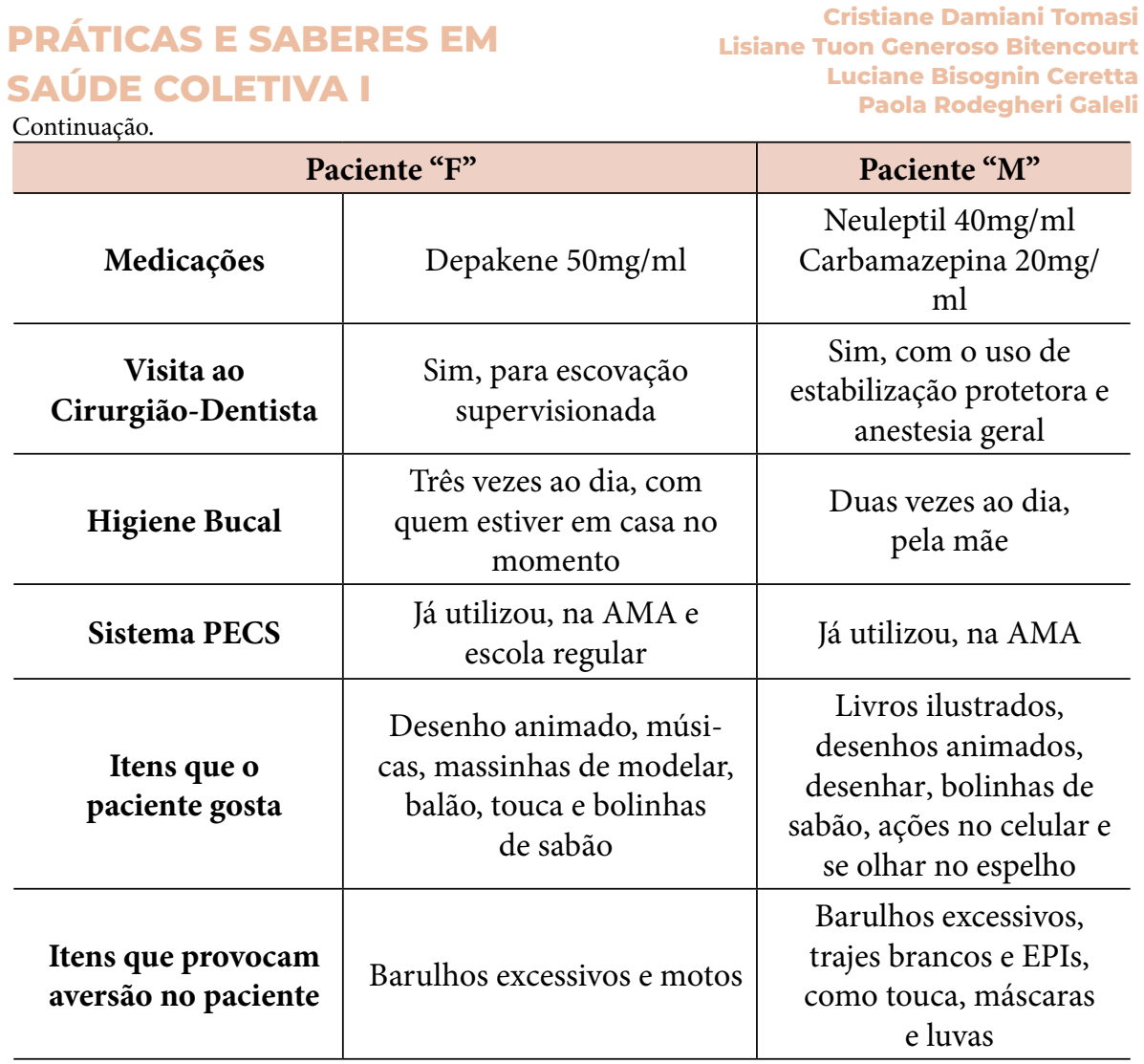

Fonte: Elaborada pelos autores.

CID: Classificação Internacional de Doenças

PECS: Picture Exchange Communication System

AMA: Associação Amigo dos Autistas

EPIs: Equipamento de Proteção Individual

No primeiro atendimento existiam duas opções para o acolhimento dos participantes, a cadeira odontológica e o tapete de Etil Vinil e Acetato (EVA). A opção de escolha entre um ou outro, para iniciar a consulta, ficou a cargo dos participantes e ambos optaram por utilizar o tapete, sendo que esta escolha permaneceu durante os atendimentos seguintes até que se sentassem na cadeira odontológica (Figura 1). 
Figura 1: Sala de Atendimento.

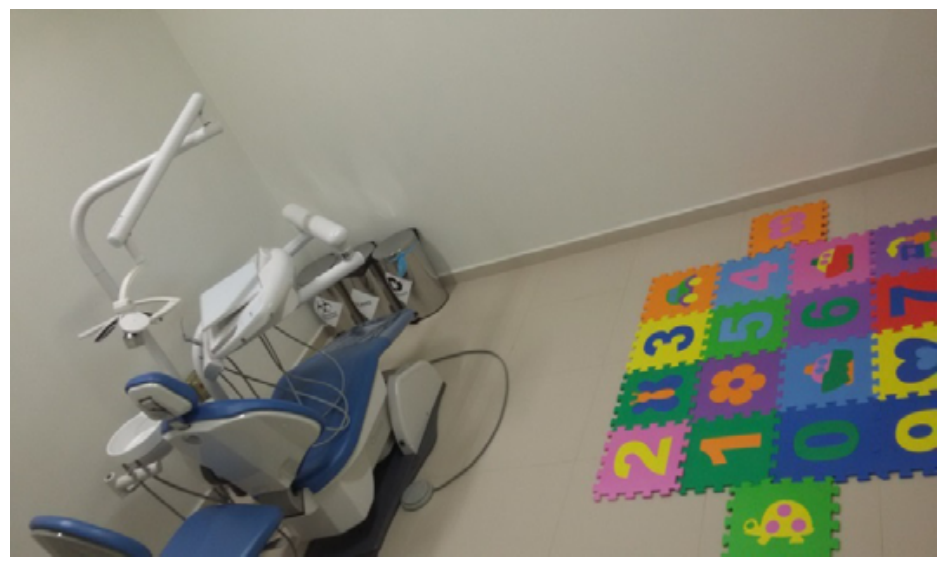

Fonte: Elaborada pelos autores.

A apresentação do PECS foi realizada por meio do método falarmostrar-fazer. Sendo que neste momento a paciente " $F$ " se mostrou atenta às explicações e conseguiu identificar as figuras e montou a sequência que foi demonstrada (Figura 2). O paciente "M" se mostrou relutante ao método, não fixou sua atenção ao que era repassado.

Figura 2: Demonstração de sete das dez figuras do modelo PECS.

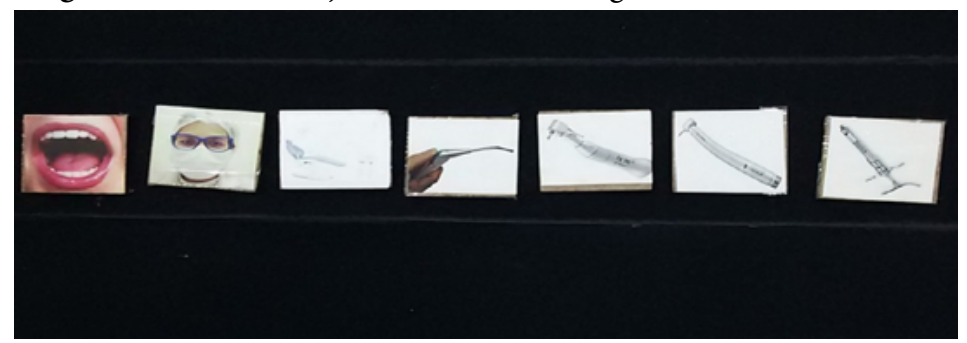

Fonte: Elaborada pelos autores.

Quanto ao uso da cadeira odontológica, houve a colaboração dos responsáveis e, deste modo, a paciente " $F$ " conseguiu sentar-se sozinha, per- 


\section{PRÁTICAS E SABERES EM

mitindo que a apresentação do PECS continuasse. Enquanto " $M$ " se sentou na cadeira, somente quando auxiliado pelo responsável, porém sem êxito quanto ao método de abordagem.

A partir da análise comportamental do paciente " $M$ ", o sistema de apresentação foi modificado, pois o mesmo se mostrava agressivo ao método de escolha inicial. Desta forma, foi confeccionado um PECS em forma de revista, contendo as mesmas figuras, devido à afinidade do paciente com esta forma de apresentação (Figura 3).

Figura 3: Apresentação PECS em forma de revista

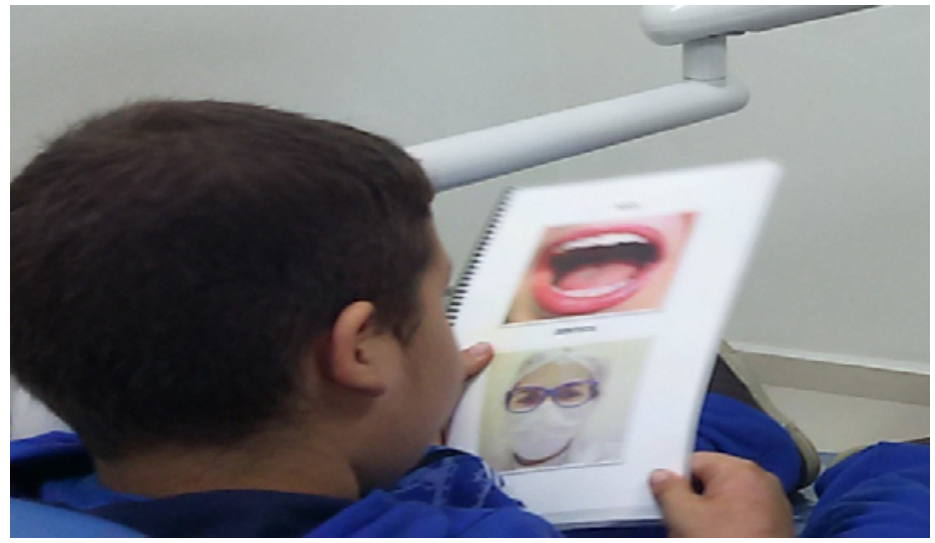

Fonte: Elaborada pelos autores.

Para a paciente "F" o tapete foi retirado a partir da segunda consulta, o que não ocorreu com " $\mathrm{M}$ ”, que necessitou de um tempo maior para a retirada do tapete de EVA.

O paciente " $M$ " conseguiu sentar-se na cadeira odontológica, mesmo que por pouco tempo. O uso da luz, sugador e tríplice foi alcançado com êxito, porém no período em que " $M$ " estava participando do estudo e sendo condicionado, ele necessitou de intervenção odontológica por outro profissio- 
nal, que foi realizada com anestesia geral, propiciando regressão no condicionamento e atenção especial posteriormente.

A paciente " $F$ " durante o condicionamento lúdico na primeira etapa do estudo utilizou todos os itens relacionados ao consultório, EPIs e procedimentos clínicos como radiografias interproximais, profilaxia com uso de curetas Gracey Quinelato ${ }^{\circledR}$ e baixa rotação com escova Robinson, taça de borracha e pasta profilática Maquira ${ }^{\circledR}$ associada a pedra-pomes. Além disso, $\mathrm{o}$ atendimento à paciente foi finalizado com aplicações de selante ionomérico Ketac Molar Easy Mix da $3 \mathrm{M}^{\circledast}$ nos primeiros molares permanentes inferiores que apresentavam pigmentações nos sulcos oclusais, sugestivos de lesão incipiente de cárie. Optou-se por selante ionomérico, pois na primeira etapa do estudo não estava prevista a utilização de anestesia local e consequentemente de isolamento absoluto.

Toda ação conquistada, durante os atendimentos, foi reforçada com palmas e palavras positivas como: "Muito bem", "Parabéns", "É isso aî". Ao final de cada consulta, os PECS eram reafirmados e remontados pela paciente "F" no quadro, para a afirmação da conquista diária. Com "M", os PECS eram revisados por meio da revista previamente elaborada.

O paciente " $M$ " não alcançou o objetivo do condicionamento lúdico durante a primeira etapa do estudo, motivo pelo qual foi encaminhado para a Clínica de Odontologia para Pacientes com Necessidades Especiais da UNESC, para dar continuidade ao condicionamento e posterior procedimentos clínicos necessários, não seguindo para a segunda etapa do estudo.

A paciente " $F$ " alcançou todos os objetivos iniciais do estudo, sendo assim foi acompanhada, pelas mesmas pesquisadoras, após seis meses do final da primeira etapa, no mesmo local de atendimento, dias e horários, para a execução dos tratamentos odontológicos necessários, com o uso ou não de anestesia local.

Após o retorno aconteceram três atendimentos e foram realizados procedimentos tais como profilaxia com uso de ultrassom Gnatus ${ }^{\oplus}$, curetas Gracey Quinelato ${ }^{\circledR}$, baixa rotação com escova Robinson, taça de borracha, 
pasta profilática Maquira ${ }^{\circledast}$ com pedra-pomes, radiografias interproximais e instrução de higiene oral. Por meio dos exames clínicos e radiográficos constatou-se ausência de lesões de cárie que necessitassem de intervenção odontológica (Figura 4).

Figura 4: Realização de procedimentos odontológicos na segunda etapa do estudo

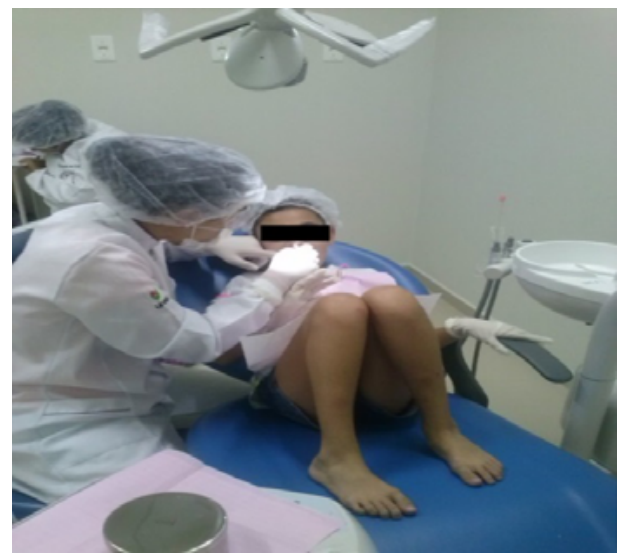

Fonte: Elaborada pelos autores.

Durante os atendimentos que ocorreram na segunda etapa do estudo, "F" cooperou, porém em alguns momentos se mostrou impaciente frente às atividades realizadas. Um dos métodos utilizados para tranquilizar a paciente foi a musicoterapia, que funcionou adequadamente e permitiu que os procedimentos fossem finalizados.

Ao decorrer da realização das radiografias " $F$ ” não permitiu que estas fossem feitas sem auxílio do responsável, que teve que acomodá-la em seu colo para facilitar o posicionamento do filme radiográfico na boca da participante. Na primeira etapa do estudo " $F$ " havia permitido a realização da tomada radiográfica para a detecção de cárie, porém as mesmas não se mostraram claras para o correto diagnóstico.

No segundo momento, com o auxílio do responsável, foi possível realizar novas radiografias interproximais e confirmar a ausência de lesões de 
cárie nos sulcos oclusais, pigmentados, dos primeiros molares permanentes inferiores, selados com Ketac Molar Easy Mix da $3 \mathrm{M}^{\oplus}$, na primeira etapa do estudo. A ausência das lesões de cárie nas radiografias pode ser devido a paralização das lesões incipientes por meio da ação do selante ionomérico aplicado na primeira etapa do estudo juntamente com a melhora da higienização bucal da paciente.

O estudo verificou a evolução de dois pacientes com TEA por meio dos PECS. Um paciente atingiu a totalidade do objetivo, enquanto o outro se encontrava em evolução. Em uma pesquisa realizada na cidade de São Paulo, que utilizou a mesma metodologia, o resultado apresentou uma diferença estatística ( $\mathrm{p}=0,006)$ na aceitação dos PECS realizado entre dois grupos (G1 e G2). No grupo G1 os participantes não tiveram experiência odontológica anterior, enquanto no grupo G2 os participantes tiveram experiência odontológica anterior. Ambos os grupos conseguiram utilizar os PECS, entretanto o artigo relata que não rejeitou a hipótese nula e que houve uma melhor aceitação pelo grupo G1 (ZINK, 2012b).

Na Itália, o comportamento social comunicativo foi avaliado através do uso de PECS versus a terapia de linguagem convencional (CLT) em pacientes com TEA. O resultado apontou que houve uma melhora na relação social das crianças que utilizaram PECS, quando comparadas com o uso da CLT (LERNA et al., 2012).

O PECS proporciona um protocolo de ensino estruturado que pode ser facilmente executado em pacientes com TEA (AGIUS; VANCE, 2015). É uma alternativa por intermédio de figuras, que visa auxiliar no ato de comunicação destes indivíduos, desde a diferenciação destas figuras, até utilizá-las para mostrar aquilo que se quer em um determinado momento (MIZAEL; AIELLO, 2013).

Estudos realizados na cidade de São Paulo-SP e em Guaratinguetá-SP, mostraram que pacientes que não tiveram experiência odontológica anterior obtiveram uma desenvoltura maior para aceitação dos PECS em comparação com aqueles que já possuíam (CALTABIANO et al., 2015; ZINK, 2012b). No 
presente estudo, a paciente " $F$ " apresentou uma facilidade na aceitação da primeira tentativa da utilização dos PECS, uma vez que sua experiência odontológica prévia foi focada na escovação supervisionada e não em procedimentos invasivos, enquanto o paciente " $M$ " apresentou maiores dificuldades, devido às experiências traumáticas vivenciadas anteriormente ao estudo.

Para que sejam alcançados resultados melhores em relação ao manejo odontológico destes pacientes é preconizado que os procedimentos sejam realizados mantendo os mesmos horários, local e auxiliar do profissional durante as consultas, e deve ser realizado um protocolo a fim de melhorar o relacionamento com o paciente (KLEIN; NOWAK, 1998; MAGALHÃES, 2006; SWALLOW, 1969; ZINK, 2012b). O tempo de espera para a consulta não deve ultrapassar quinze minutos e o cirurgião-dentista deve evitar falar termos técnicos próximo ao paciente (KLEIN; NOWAK, 1998). No presente estudo, os pacientes reconheciam e guiavam os responsáveis até o local onde as consultas eram realizadas uma vez que os horários, local, pesquisadora auxiliar e tempo de espera foram respeitados.

No presente estudo foi observado que os participantes melhoraram a comunicação com as pesquisadoras. Esta conquista foi crescente ao decorrer das consultas, tendo uma melhora no relacionamento paciente e profissional. Outros estudos realizados com pacientes diagnosticados com TEA relataram que houve uma evolução dos hábitos comportamentais e na comunicação quando se aplicou este método (FERREIRA; CIELO; TREVISAN, 2010; ZINK, 2012b). Por outro lado, Caltabiano e colaboradores (2015), evidenciaram um maior obstáculo quanto ao uso do condicionamento lúdico em pacientes com TEA devido à dificuldade na comunicação (CALTABIANO et al., 2015). Crianças diagnosticadas com TEA possuem uma melhor comunicação por meio de imagens do que utilizando palavras. Desta forma a psicologia visual facilita a compreensão de novos aprendizados, principalmente para a introdução da Odontologia na vida destes pacientes (DELLI et al., 2013) (PILEBRO; BACKMAN, 2005).

O uso do condicionamento lúdico é incentivado em pacientes com TEA na Odontologia, antes de qualquer intervenção hospitalar (ZINK, 2012b), 
visto que procedimentos realizados em âmbito hospitalar podem produzir estresse e aborrecimentos. Porém, percebe-se que os responsáveis de crianças com TEA possuem maior aceitação com a de anestesia geral (MARSHALL et al., 2008). No presente estudo, o paciente " $M$ ", sofreu uma intervenção odontológica com uso de anestesia geral no transcorrer da pesquisa. Tal fato contribuiu para a regressão do seu condicionamento lúdico, motivo pelo qual o paciente acabou sendo excluído da segunda etapa da pesquisa.

Os pacientes na consulta inicial preferiram o primeiro contato por meio do tapete de EVA, onde ambos demonstraram receio de sentar-se na cadeira odontológica, podendo ser justificado pela experiência odontológica anterior ou medo do desconhecido. Na literatura há resultado semelhante ao encontrado, uma vez que os pacientes que já tiveram contato odontológico anterior preferiram iniciar a consulta sentados no chão(ZINK, 2012b).

As técnicas mais abordadas para o auxílio do condicionamento lúdico são falar-mostrar-fazer, reforço positivo, verbal diferenciado, erradicação de estímulos sensoriais que desencadeiam o estresse, comandos claros e objetivos e estabelecimento de uma rotina de atendimento (DELLI et al., 2013; ZINK, 2012 b). No presente estudo, as técnicas mais utilizadas foram a do falar-mostrar-fazer e reforçadores positivos.

$\mathrm{O}$ uso de reforçadores é um meio importante para o atendimento de pacientes com TEA, pois auxilia na recompensa para o alcance dos objetivos propostos. Há diversas formas de utilizar os reforçadores positivos, como por exemplo presentes e palavras de motivação que auxiliam a cooperação do paciente no âmbito odontológico (DELLI et al., 2013). No presente estudo, os reforçadores utilizados foram palavras motivacionais que auxiliaram na evolução dos pacientes.

Klein e colaboradores (1998), afirmam que a participação dos responsáveis durante o atendimento não é indicada (KLEIN; NOWAK, 1998). No presente estudo, a presença dos familiares facilitou durante as primeiras consultas e para a adaptação dos pacientes no âmbito odontológico. Outros relatos corroboram esta afirmação e demonstram que incluir a presença dos 
pais durante o atendimento odontológico é um método básico que auxilia profissionais com pacientes com TEA (CALTABIANO et al., 2015; DELLI et al., 2013).

Pacientes que alcançam uma nova habilidade durante o tratamento odontológico por meio do PECS, não retrocedem nos objetivos conquistados (CALTABIANO et al., 2015; ZINK, 2012b). Isto condiz com o que foi encontrado na segunda etapa do presente estudo onde a paciente "F" permaneceu condicionada mesmo após um período de recesso nos atendimentos odontológicos, porém o mesmo não ocorreu com o paciente " $M$ ", pois ele involuiu após os procedimentos realizados em âmbito hospitalar, impossibilitando o encaminhamento dele para a segunda etapa do estudo.

Em alguns momentos se utilizou musicoterapia por meio do canto, que auxiliou durante os atendimentos, tranquilizando a paciente. Este método utiliza o som como meio de interação e comunicação e pode ser realizado por meio do canto, movimentos a partir do estímulo sonoro e escuta de melodias (BRASIL, 2000).

É de suma importância que o atendimento odontológico para pacientes com TEA seja individualizado e que suas alterações comportamentais sejam levadas em consideração. Estes pacientes necessitam de atendimento especializado e compreensão de suas necessidades. Cada indivíduo responde de maneira diferente, sendo necessária a intervenção que promova e previna a saúde, além de educação continuada e permanente dos profissionais e responsáveis a fim de melhorar a qualidade de vida destes pacientes (ALVES, 2004; BRASIL, 2000; CHEW; KING; ODONNELL, 2006; DELLI et al., 2013; MAGALHÃES, 2006; ZINK, 2012b).

Ressalta-se que existem poucos trabalhos publicados que abordem como deve ser realizado o atendimento de pacientes autistas em âmbito odontológico (AMARAL; PORTILHO; MENDES, 2011; JAGANATHAN, 2014; KATZ et al., 2009; MAREGA; AIELLO, 2005). Sugere-se que novos estudos sejam realizados com um número maior de pacientes com TEA, visando afir- 
mar cada vez mais a eficiência do método do condicionamento lúdico com o auxílio dos PECS.

\section{CONCLUSÃO}

É indispensável um olhar holístico para com os pacientes portadores de necessidades especiais. O uso dos PECS, neste atendimento, facilita a comunicação com o paciente; porém, é necessário dedicação e paciência para a realização dos procedimentos.

O condicionamento lúdico no atendimento odontológico de crianças com diferentes graus de TEA auxilia na prática odontológica e facilita a comunicação profissional e paciente.

A pesquisa serve como base para o atendimento diferenciado dos pacientes com TEA; o método, porém, pode ser utilizado em pacientes com outras necessidades especiais e até mesmo com pessoas que sofrem ansiedade no ambiente odontológico.

\section{REFERÊNCIAS}

AGIUS, May M.; VANCE, Margaret. A Comparison of PECS and iPad to Teach Requesting to Pre-schoolers with Autistic Spectrum Disorders. Augmentative And Alternative Communication, [s.l.], v. 32, n. 1, p. 58-68, 20 nov. 2015.

ALVES, Elaine Gomes dos Reis. A Singularidade do atendimento odontológico a pacientes portadores de Síndrome de Autismo. JSO. [s. L.]. fev. 2004. Disponível em: <http://www.jornaldosite.com.br/arquivo/anteriores/elaine/ artelaine81.htm>. Acesso em: 14 maio 2016.

AMARAL, Lais David; PORTILHO, Jorge Alberto Cordón; MENDES, Silvia Carolina Teixeira. Estratégias de acolhimento e condicionamento do paciente autista na Saúde Bucal Coletiva. Tempus Actas de Saúde Coletiva, Brasília, v. 
5, n. 3, p.105-114, jan. 2011. Disponível em: <http://www.tempusactas.unb.br/ index.php/tempus/article/view/1046/955>. Acesso em: 8 mar. 2017.

BRASIL. MINISTÉRIO DA SAÚDE. Autismo: orientação para os pais. Brasília, 2000. 37 p. Disponível em: <http://bvsms.saude.gov.br/bvs/publicacoes/ cd03_14.pdf $>$. Acesso em: 8 mar. 2017.

CALTABIANO, Rosângela Monteiro et al. Estudo e atendimento a pacientes especiais com proposta diferenciada de adaptação e condicionamento em consultório odontológico. In: $8^{\circ}$ CONGRESSO DE EXTENSÃO UNIVERSITÁRIA DA UNESP, 8., 2015, [s.l.]. Anais... [s.l.]: Unesp, 2015. p. 1-7. Disponível em: <https://repositorio.unesp.br/handle/11449/142439>. Acesso em: 14 ago. 2016.

CHEW, Leslie C T; KING, Nigel M; O’DONNELL, David. Autism: the Aetiology, Management and Implications for Treatment Modalities from the Dental Perspective. Dental Update, [s.1.], v. 33, n. 2, p. 70-83, 2 mar. 2006.

CHRISTENSEN, Deborah L. et al. Prevalence and Characteristics of Autism Spectrum Disorder Among Children Aged 8 Years - Autism and Developmental Disabilities Monitoring Network, 11 Sites, United States, 2012. Mmwr. Surveillance Summaries, [s.1.], v. 65, n. 3, p. 1-23, 2016.

DELLI, K. et al. Management of children with autism spectrum disorder in the dental setting: Concerns, behavioural approaches and recommendations. Medicina Oral Patología Oral y Cirugía Bucal, [s.l.], p. 862-868, 2013. Disponível em: <https://core.ac.uk/download/pdf/33067861.pdf > . Acesso em: 8 mar. 2017.

DIAS, Sandra. Asperger e sua síndrome em 1944 e na atualidade. Revista Latinoamericana de Psicopatologia Fundamental, [s.l.], v. 18, n. 2, p. 307313, jun. 2015. FapUNIFESP (SciELO). Disponível em: <http://www.scielo.br/ pdf/rlpf/v18n2/1415-4714-rlpf-18-2-0307.pdf>. Acesso em: 30 abr. 2016.

FERREIRA, Fernanda Vargas; CIELO, Carla Aparecida; TREVISAN, Maria Elaine. Aspectos respiratórios, posturais e vocais da Doença de Parkinson: 
considerações teóricas. Revista Cefac, [s.l.], v. 13, n. 3, p.534-540, 10 dez. 2010. Disponível em: <http://www.scielo.br/scielo.php?pid=S1516$18462011000300020 \&$ script=sci_abstract\&tlng=pt $>$. Acesso em: 8 mar. 2017.

JAGANATHAN, Udhya. Autism Disorder (AD): An Updated Review for Paediatric Dentists. Journal Of Clinical And Diagnostic Research, [s.l.], p.275-279, 2014. JCDR Research and Publications. Disponível em: <https:// www.ncbi.nlm.nih.gov/pmc/articles/PMC3972586/>. Acesso em: 30 abr. 2016. KATZ, Cíntia Regina Tornisiello et al. Abordagem psicológica do paciente autista durante o atendimento odontológico. Odontologia Clín.-cient., Recife, v. 8 , n. 2, p. 115-121, abr./jun. 2009.

KLEIN, Ulrich; NOWAK, Arthur J. Autistic disorder: a review for the pediatric dentist. Pediatric Dentistry, [s. 1.], v. 20, n. 5, p. 312-317, out. 1998.

LERNA, Anna et al. Social-communicative effects of the Picture Exchange Communication System (PECS). In: Autism Spectrum Disorders. International Journal Of Language \& Communication Disorders, [s.l.], v. 47, n. 5, p. 609617, 18 jul. 2012.

MAGALHÃES, Marina Helena Cury Gallottini de. Odontologia para Pacientes Especiais: Atendimento odontológico a autistas. 2006. Disponível em: <http://www.wwow.com.br/portal/revista/revista. asp secao $=5 \&$ view $=$ artigos\&id $=112>$. Acesso em: 8 mar. 2017.

MAREGA, Tatiane; AIELLO, Ana Lúcia Rossito. Autismo e tratamento odontológico: algumas considerações. Revista Íbero-Americana de Odontopediatria e Odontologia do Bebê, Curitiba, v. 8, n. 42, p. 150-157, 2005.

MARSHALL, Jennifer et al. Parental Attitudes Regarding Behavior Guidance of Dental Patients with Autism. Pediatric Dentistry, [s.l.], v. 30, n. 5, p. 400-407, out. 2008. Disponível em: <https://pdfs.semanticscholar. org/8ae1/45af74792a864e3b2e7b843f940539426b2b.pdf>. Acesso em: 13 mar. 2017. 
MIZAEL, Táhcita Medrado; AIELLO, Ana Lúcia Rossito. Revisão de estudos sobre o Picture Exchange Communication System (PECS) para o ensino de linguagem a indivíduos com autismo e outras dificuldades de fala. Revista Brasileira de Educação Especial, Marília, v. 19, n. 4, p. 623-636, dez. 2013. Disponível em: <http://www.scielo.br/pdf/rbee/v19n4/v19n4a11.pdf > . Acesso em: 14 jul. 2016.

ORGANIZAÇÃO MUNDIAL DE SAÚDE (OMS). Classificação Estatística Internacional de Doenças e Problemas Relacionados à Saúde (CID-10). 10. ed. São Paulo: Editora da Universidade de São Paulo, 2008. v. 1. Disponível em: <http://www.datasus.gov.br/cid10/v2008/cid10.htm>. Acesso em 6 mar. 2016. ORIQUI, Maria Sílvia Younes. Avaliação clínica das condições de saúde bucal de pacientes autistas. 2006. 57 f. Dissertação (Mestrado) - Curso de Pós-graduação em Ciências da Saúde, Faculdade de Medicina de São José do Rio Preto, São José do Rio Preto, 2006. Disponível em: <http://bdtd.famerp.br/bitstream/ tede/213/1/mariasilviaoriqui_dissert.pdf $>$. Acesso em: 14 maio 2016.

PILEBRO, C.; BACKMAN, B. Teaching oral hygiene to children with autism. International Journal Of Paediatric Dentistry, [s.1.], v. 15, n. 1, p. 1-9, jan. 2005.

SWALLOW, James Neil. The dental management of autistic children. British Dental Journal, [s.l], v. 126, n. 3, p. 128-131, fev. 1969.

ZINK, Adriana. Com ou sem anestesia geral? Revista Autismo, [s. L.], n. 2, p.11-11, abr. 2012a. Disponível em: <http://www.revistaautismo.com.br/ RevistaAutismo002.pdf>. Acesso em: 13 mar. 2016.

ZINK, Adriana Gledys. Uso do sistema por intercâmbio de figuras (PECS) para o tratamento odontológico de indivíduos com autismo. 2012.136f. Dissertação (Mestrado) - Curso de Mestrado em Ciências da Saúde, Universidade Cruzeiro do Sul, São Paulo, 2012b. 